\title{
AGE AND SEX SPECIFIC RISK PROFILES AND IN-HOSPITAL OUTCOME IN 2,573 SUBJECTS FROM A MULTICENTRE COHORT
}

D. Vázquez ${ }^{1}$, S. Joaquin ${ }^{2}$, I. Benabdelhak ${ }^{1}$, A. Vena ${ }^{1}$, A.M. de Arce ${ }^{3}$, G. Mauri-Capdevila ${ }^{1}$, J. Sanahuja ${ }^{1}$, B. Fuentes ${ }^{4}$, M. Millan ${ }^{5}$, J.F.

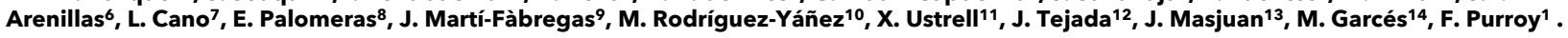
${ }^{1}$ Hospital Arnau de Vilanova, Stroke Unit, Lleida, Spain. ${ }^{2}$ Hospital Dr. Josep Trueta., Stroke Unit, Girona, Spain. ${ }^{3}$ Hospital de Donostia, Stroke Unit, San Sebastián, Spain. ${ }^{4 H}$ Hospital de la Paz, Stroke Unit, Madrid, Spain. ${ }^{5}$ Hospital Trias i Pujol, Stroke Unit, Badalona, Spain. ${ }^{6}$ Hospital Clinico, Stroke Unit, Valladolid, Spain. ${ }^{7}$ Hospital Moisés Broggi, Stroke Unit, Esplugues de Llobregat, Spain. ${ }^{8} \mathrm{Hospital}$ de Mataró, Neurology Department, Mataró Spain. ${ }^{9}$ Hospital de la Santa Creu i Sant Pau, Stroke Unit, Barcelona, Spain. ${ }^{10} \mathrm{Hospital}$ Universitario de Santiago de Compostela, Stroke Unit, Santiago de Compostela, Spain. ${ }^{11} \mathrm{Hospital} J$ Joan XXIII, Neurology, Tarragona, Spain. ${ }^{12}$ Hospital de León, Neurology, Leon, Spain. ${ }^{13}$ Hospital Ramón y Cajal, Stroke Unit, Madrid, Spain. ${ }^{44}$ Hospital de Tortosa, Stroke Unit, Tortosa, Spain

\section{INTRODUCTION:}

We aimed to determine the age and sex related patterns and predictors of the clinical course in a large multi-centre registry (from Spain's nationwide hospital-based stroke registry, RENISEN) of admitted haemorrhagic stroke (HS) patients.

Clinical characteristics by age groups among hemorrhagic stroke admissions

\begin{tabular}{|c|c|c|c|c|c|c|c|c|c|}
\hline & & & & & Age groups & & & & \\
\hline Total n.\%) & $\frac{\text { All }}{257}$ & $\frac{18.39}{57}$ & $\begin{array}{l}40-49 \\
134\end{array}$ & $\begin{array}{l}50.59 \\
529\end{array}$ & $60-69$ & $\begin{array}{l}70-79 \\
806\end{array}$ & $80-89$ & 290 & \\
\hline $\begin{array}{l}\text { Age, years } \\
\text { Ages }\end{array}$ & $71.4(13.1)$ & $33.8(5.1)$ & $\begin{array}{l}134.09 \\
44.9(3.0)\end{array}$ & $54.8(2.9)$ & $\begin{array}{r}482.9 \\
65.0(2.9)\end{array}$ & $\begin{array}{l}806.0(2.8) \\
\end{array}$ & $\begin{array}{l}734 \\
83.5(2.6)\end{array}$ & $\begin{array}{c}78 \\
91.9(2.0)\end{array}$ & p-value \\
\hline \multicolumn{10}{|l|}{$\begin{array}{l}\text { Baseline } \\
\text { characteristics }\end{array}$} \\
\hline $\begin{array}{l}\text { female sex } \\
\text { Femate }\end{array}$ & $1005(39.1)$ & $29(50.9)$ & & & $141(29.3)$ & $305(37.8)$ & $368(50.1)$ & & \\
\hline Alcoholism & $141(5.5)$ & $\begin{array}{l}0(0) \\
0(0)\end{array}$ & $15(10.6)$ & 42 (14.9) & $\begin{array}{l}49(10.2) \\
\text { (1) }\end{array}$ & $28(3.5)$ & $6(0.8)$ & $1(1.3)$ & $<0.001$ \\
\hline Smoker & $310(12.0)$ & $15(26.3)$ & $48(35.8)$ & $84(29.8)$ & $89(18.5)$ & $51(6.3)$ & $21(2.9)$ & $2(2.6)$ & $<0.001$ \\
\hline $\begin{array}{l}\text { Hypertension } \\
\text { Pithets }\end{array}$ & $1755(68.2)$ & $15(26.3)$ & 65 (48.5) & $\begin{array}{l}161(57.1) \\
54(191)\end{array}$ & $331(68.7)$ & $568(70.5)$ & $553(75.3)$ & $62(79.5)$ & $<0.001$ \\
\hline $\begin{array}{l}\text { Diabetes melilus } \\
\text { Hyperlipidemia }\end{array}$ & $\begin{array}{l}607(23.6) \\
1009(39.2)\end{array}$ & $\begin{array}{l}11.8) \\
1(1.8)\end{array}$ & $\begin{array}{l}996(6.7) \\
23(17.2)\end{array}$ & $\begin{array}{l}54(19.1) \\
89(31.6)\end{array}$ & $\begin{array}{l}134(27.8) \\
221(45.9)\end{array}$ & $\begin{array}{l}222(1727.4) \\
358(444.4)\end{array}$ & $\begin{array}{l}177(23.8) \\
290(39.5)\end{array}$ & $\begin{array}{l}133(16.7) \\
27(34.6)\end{array}$ & $\begin{array}{l}<0.001 \\
<0.001\end{array}$ \\
\hline Atrial fibrillation & $324(12.6)$ & $\begin{array}{l}0(1.8) \\
0(0)\end{array}$ & $\begin{array}{l}2(1.5) \\
2(17 .)\end{array}$ & $\begin{array}{l}89(1.5) \\
7(2.5)\end{array}$ & $28(5.8)$ & $\begin{array}{l}3180(144.4) \\
113(14.0)\end{array}$ & $160(21.8)$ & $\begin{array}{l}14(17.9) \\
14.96)\end{array}$ & $<0.001$ \\
\hline $\begin{array}{l}\text { Ischemic heart } \\
\text { disease }\end{array}$ & $190(7.4)$ & $0(0)$ & $1(0.7)$ & $12(4.3)$ & $31(6.4)$ & $76(9.4)$ & $60(8.2)$ & $10(12.8)$ & $<0.001$ \\
\hline $\begin{array}{l}\text { Previous ischemic } \\
\text { stroke }\end{array}$ & $243(9.4)$ & $0(0)$ & $1(0.7)$ & $15(5.3)$ & $37(7.7)$ & $97(12.0)$ & $86(11.7)$ & $7(9.0)$ & $<0.001$ \\
\hline $\begin{array}{l}\begin{array}{l}\text { Previous } \\
\text { hemorrthagic } \\
\text { stroke }\end{array}\end{array}$ & $197(7.7)$ & $4(7.0)$ & $5(3.7)$ & $13(4.6)$ & $33(6.8)$ & $80(9.9)$ & $57(7.8)$ & $5(6.4)$ & 0.041 \\
\hline \multicolumn{10}{|l|}{$\begin{array}{l}\begin{array}{l}\text { Previous } \\
\text { treatment }\end{array} \\
\text {. }\end{array}$} \\
\hline Anticoagulants & $419(16.3)$ & $0(0)$ & $2(1.5)$ & $17(6.0)$ & $51(10.6)$ & $167(20.7)$ & $173(23.6)$ & $9(11.5)$ & $<0.001$ \\
\hline 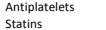 & $\begin{array}{l}687(26.7) \\
823(32.0)\end{array}$ & $\begin{array}{l}1(1.8) \\
2(3.5)\end{array}$ & $\begin{array}{l}5(3.7) \\
13(9.7) \\
\end{array}$ & $\begin{array}{l}\begin{array}{l}43(15.2) \\
56(119.9)\end{array}\end{array}$ & $\begin{array}{l}116(24.1) \\
177(36.7)\end{array}$ & $\begin{array}{l}248(30.8) \\
305(37.8)\end{array}$ & $\begin{array}{l}241132.8) \\
24(33.9)\end{array}$ & $\begin{array}{l}33(42.3) \\
21(26.9)\end{array}$ & $\begin{array}{l}<<.001 \\
<0.001\end{array}$ \\
\hline Antihypertensive & $1444(56.1)$ & ${ }_{10}(17.5)$ & $42(31.3)$ & 114(40.9) & 273.56 .65 & (99067.08 & $464(63.27)$ & 51(65) & $<0.001$ \\
\hline S & 478 (18.6) & $0(0)$ & $6(45)$ & 年 & (1) & 43000.01 & 等 & (1) & . \\
\hline \multicolumn{10}{|l|}{ Management } \\
\hline $\begin{array}{l}\text { Stroke code } \\
\text { activation }\end{array}$ & $1324(51.5)$ & $31(54.4)$ & $67(50.0)$ & $158(56.0)$ & $264(54.8)$ & $432(53.6)$ & $349(47.5)$ & $23(29.5)$ & 0.003 \\
\hline $\begin{array}{l}\text { Stroke unit } \\
\text { admission }\end{array}$ & $1689(65.6)$ & $39(68.4)$ & $89(66.4)$ & $217(77.0)$ & $331(68.7)$ & $538(66.7)$ & $445(60.6)$ & $30(38.5)$ & $<0.001$ \\
\hline Intensive care & $208(8.1)$ & $12(21.1)$ & $19(14.2)$ & $34(12.1)$ & $62(12.9)$ & $63(7.8)$ & $16(2.2)$ & $2(2.6)$ & $<0.001$ \\
\hline \multicolumn{10}{|l|}{ Stroke severity } \\
\hline Basal mRS & $0.0(0.0-2.0)$ & $0.0(0.0-0.0 .0)$ & $\begin{array}{l}0.0(0.0-0.0) \\
\end{array}$ & $0.0(0.0-0.0)$ & $0.0(0.0-0.0)$ & $\begin{array}{l}0.0(0.0-0.0) \\
\end{array}$ & $1.0(0.0-3.0)$ & $2.0(0.0-3.0)$ & $<0.001$ \\
\hline $\begin{array}{l}\text { NIHSS at } \\
\text { admission }\end{array}$ & $7.0(3.0-16.0)$ & $\begin{array}{l}3.0(1.0- \\
\text { i10 }\end{array}$ & $\begin{array}{l}5.011 .0- \\
13.5\end{array}$ & $\begin{array}{l}6.012 .0- \\
14.0\end{array}$ & $\begin{array}{l}7.013 .0- \\
.150 .\end{array}$ & $\begin{array}{l}7.0(3.0- \\
160\end{array}$ & $\begin{array}{l}7.013 .0- \\
0160\end{array}$ & $\begin{array}{l}9.013 .0- \\
.180 .\end{array}$ & $<0.001$ \\
\hline \multicolumn{10}{|l|}{$\begin{array}{l}\text { admission } \\
\text { Etiology }\end{array}$} \\
\hline Primary & 1955 (76.0) & $23(40.4)$ & $88(65.7)$ & $208(73.8)$ & $382(79.3)$ & $614(76.2)$ & $574(78.2)$ & 66 (884.6) & $<0.001$ \\
\hline Secondary & $488(19.0)$ & $25(43.9)$ & $31(23.1)$ & $57(20.2)$ & $82(17.0)$ & $148(18.4)$ & $136(18.5)$ & $9(11.5)$ & \\
\hline Unknown & & & & & & & $\begin{aligned} 24(3.3) \\
3290(153)\end{aligned}$ & & 5001 \\
\hline $\begin{array}{l}\text { Hypertensive } \\
\text { Amyloidal }\end{array}$ & $\begin{array}{l}\begin{array}{l}1448(56.3) \\
342(13.3)\end{array} \\
\end{array}$ & $\begin{array}{c}\begin{array}{c}19(33.3) \\
0(0)\end{array} \\
0\end{array}$ & $\begin{array}{l}77(175.5) \\
0(0)\end{array}$ & 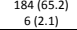 & $\begin{array}{l}323(67.0) \\
30(6.2)\end{array}$ & $\begin{array}{l}411(51.0) \\
140(17.4)\end{array}$ & $\begin{array}{l}392(53.4) \\
148(20.2)\end{array}$ & $\begin{array}{l}\begin{array}{l}42(55.38) \\
18(23.1)\end{array} \\
\end{array}$ & $<0.001$ \\
\hline \multicolumn{10}{|l|}{$\frac{\text { Anyloidal }}{\text { ICH location }}$} \\
\hline & $922(35.8)$ & $19(33.3)$ & $39(29.1)$ & $73(25.9)$ & $136(28.2)$ & $329(40.8)$ & $290(39.5)$ & $36(46.2)$ & $<0.001$ \\
\hline Deep & 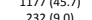 & $\begin{array}{l}23(40.4) \\
132028\end{array}$ & $\begin{array}{l}70(52.2) \\
15(21)\end{array}$ & 145351.47) & $264(54.8)$ & $\begin{array}{l}343(42.6) \\
4(42.6)\end{array}$ & $\begin{array}{l}3000(40.9) \\
\end{array}$ & 32(241.0) & \\
\hline (V) & 年 & 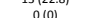 & 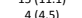 & $\begin{array}{lll} & \end{array}$ & (10.1) & (24) & 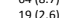 & 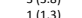 & \\
\hline Unkonwn & $175(6.8)$ & $2(3.5)$ & $\begin{array}{l}6(4.5) \\
6(4.5)\end{array}$ & $22(7.8)$ & 28.87 & $\begin{array}{l}50(6.2) \\
50.21\end{array}$ & $\begin{array}{l}618.31 \\
618.31\end{array}$ & 667.7 & \\
\hline \multicolumn{10}{|l|}{ Clinical evolution } \\
\hline Febrile syndrome & $643(25.0)$ & $9(15.8)$ & $17(12.7)$ & $42(14.9)$ & $111(23.0)$ & $218(27.0)$ & $224(30.5)$ & $22(28.2)$ & $<0.001$ \\
\hline Re & $422(16.4)$ & $4(7.0)$ & $12(9.0)$ & $28(9.9)$ & $73(15.1)$ & $140(17.4)$ & $151(20.6)$ & $14(17.9)$ & $<0.001$ \\
\hline $\begin{array}{l}\text { Urinary infection } \\
\text { Una }\end{array}$ & $217(8.4)$ & $5(8.8)$ & 6 & $13(4.6)$ & $37(7.7)$ & $79(9.8)$ & $69(9.4)$ & $8(10.3)$ & 0.072 \\
\hline mRS at discharge & $4.0(2.0-5.0)$ & $2.0(1.0-3.0)$ & $2.0(1.0-4.0)$ & $3.0(1.0-4.0)$ & $3.0(1.0-5.0)$ & $4.0(2.0-5.0)$ & $4.0(3.0-5.50)$ & $5.0(3.0-6.0)$ & $<0.001$ \\
\hline misszz al & $1690(68.5)$ & $20(35.7)$ & $52(39.7)$ & $154(55.6)$ & $280(61.0)$ & $549(71.1)$ & $571(81.6)$ & 64(88.9) & $<0.001$ \\
\hline $\begin{array}{l}\text { Death } \\
\text { Death }\end{array}$ & $393(15.3)$ & $1(1.8)$ & $5(3.7)$ & $14(5.0)$ & $41(8.5)$ & $125(15.5)$ & $183(24.9)$ & $24(30.8)$ & $<0.001$ \\
\hline $\begin{array}{l}\text { Hos } \\
\text { fos }\end{array}$ & $7.0(4.0-12.0)$ & 8.0 & $\begin{array}{l}8.015 .0 \\
11001\end{array}$ & $\begin{array}{l}8.055 .0 \\
1.01 \\
110)\end{array}$ & $8.015 .0-$ & 7.0(4.0- & $7.014 .0-$ & $6.0(2.0-$ & $<0.001$ \\
\hline
\end{tabular}

\section{RESULTS:}

The mean (SD) age was 71.2 (13.2) years. $964(39.1 \%)$ subjects were women and $772(31.3 \%)$ were $>80$ years old. $1690(68.5 \%)$ had $\mathrm{mRs}>2$ at discharge. Older patients were more likely to be female and exhibit primary aetiologies. Previous treatment with anticoagulants was more frequent in women than in men. Older subjects and women were less likely to be admitted to a stroke unit or have a stroke code activated. Age, female sex diabetes mellitus, NIHSS at admission and febrile syndrome were predictors of poor outcome.

\section{CONCLUSION:}

Clinical characteristics, aetiology and management differed with respect to age group and sex. The poorest outcome was in elderly patients. Strategies need to be implemented to ensure equal care was given to all age groups and sex.

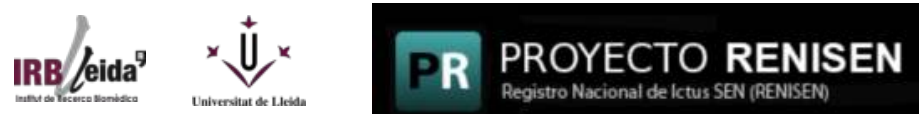

METHODS:

We prospectively studied 2,573 HS from 19 university hospitals. Post traumatic intracranial hemorrhage, post-fybrinlosis HS and subarachnoid hemorrhage were excluded. data were analysed by age groups: 18 to 39,40 to 49,50 to 59,60 to 69,70 to 79 , 80 to 89 and $\geq 90$ years). Significant disability was defined as a modified Rankin score $(\mathrm{mRS})$ at discharge $>2$.

\begin{tabular}{|c|c|c|c|c|}
\hline & All & Sex male & Sex female & p-value \\
\hline Total, $n$ (\%) & 2573 & $1568(60.94)$ & $1005(39.05)$ & \\
\hline Age, years (DE) & $71.4(13.1)$ & $69.92(12.9)$ & $73.6(13.2)$ & $<0.001$ \\
\hline \multicolumn{5}{|l|}{ Baseline characteristics } \\
\hline & $141(5.5)$ & $128(8.2)$ & $13(1.3)$ & $<0.001$ \\
\hline Smoker & $310(12.0)$ & $246(15.7)$ & $64(6.4)$ & $<0.001$ \\
\hline Hypertension & $1755(68.2)$ & $1058(67.5)$ & $697(69.4)$ & 0.102 \\
\hline Diabetes mellitus & $607(23.6)$ & $396(25.3)$ & $211(21.0)$ & 0.39 \\
\hline Hyperlipidemia & $1009(39.2)$ & $625(39.9)$ & $384(38.2)$ & 0.813 \\
\hline Atrial fibrillation & $324(12.6)$ & $180(11.5)$ & $144(14.3)$ & 0.034 \\
\hline Ischemic heart disease & $190(7.4)$ & $146(9.3)$ & $44(4.4)$ & $<0.001$ \\
\hline $\begin{array}{l}\text { Previous ischemic } \\
\text { stroke }\end{array}$ & $243(9.4)$ & $161(10.3)$ & $82(8.2)$ & 0.074 \\
\hline $\begin{array}{l}\text { Previous hemorrhagic } \\
\text { stroke }\end{array}$ & $197(7.7)$ & $115(7.3)$ & $82(8.2)$ & 0.443 \\
\hline \multicolumn{5}{|l|}{ Previous treatment } \\
\hline Anticoagulants & $419(16.3)$ & 236(15.1) & $183(18.2)$ & 0.034 \\
\hline Antiplatelets & $687(26.7)$ & $467(29.8)$ & $220(21.9)$ & $<0.001$ \\
\hline Statins & $823(32.0)$ & $526(33.5)$ & $297(29.6)$ & 0.034 \\
\hline Anthyppertensives & $1444(56.1)$ & $859(54.8)$ & $585(58.2)$ & 0.088 \\
\hline Antidiabetics & $478(18.6)$ & $314(20.0)$ & $164(16.3)$ & 0.018 \\
\hline \multicolumn{5}{|l|}{ Management } \\
\hline Stroke code activation & $1324(51.5)$ & $852(54.3)$ & $472(47.0)$ & $<0.001$ \\
\hline Stroke unit admission & 1689 (65.6) & $1074(68.5)$ & $615(61.2)$ & \\
\hline Intensive care Unit & $208(8.1)$ & $135(8.6)$ & $73(7.3)$ & \\
\hline \multicolumn{5}{|l|}{ Stroke severity } \\
\hline Basal mRS & $0.0(0.0-2.0)$ & $0.0(0.0-1.0)$ & $0.0(0.0-2.0)$ & $<0.001$ \\
\hline NIHSS at admission & $7.0(3.0-16.0)$ & $7.0(3.0-16.0)$ & $8.0(3.0-17.0)$ & 0.111 \\
\hline \multicolumn{5}{|l|}{ Etiology } \\
\hline Primary & $1955(76.0)$ & $1212(77.3)$ & $743(73.9)$ & 0.106 \\
\hline Secondary & $488(19.0)$ & $277(17.7)$ & $211(21.0)$ & \\
\hline Unknown & $130(5.1)$ & $79(5.0)$ & 51 (5.1) & \\
\hline Hypertensive & $1448(56.3)$ & 939 (59.9) & $509(50.6)$ & $<0.001$ \\
\hline & $342(13.3)$ & $168(10.7)$ & $174(17.3)$ & \\
\hline \multicolumn{5}{|l|}{ ICH location } \\
\hline Lobar & $922(35.8)$ & $510(32.5)$ & $412(41.0)$ & $<0.001$ \\
\hline - Deep & $1177(45.7$ & $780(49.7)$ & $397(39.5)$ & \\
\hline - Cerebellar & $232(9.0)$ & $135(8.6)$ & $97(9.7)$ & \\
\hline - IVH & $67(2.6)$ & $38(2.4)$ & $29(2.9)$ & \\
\hline Unknown & $175(6.8)$ & $105(6.7)$ & $70(7.0)$ & \\
\hline \multicolumn{5}{|l|}{ Clinical evolution } \\
\hline Febrile syndrome & $643(25.0)$ & $394(25.1)$ & $249(24.8)$ & 0.841 \\
\hline Respiratory infection & $422(16.4)$ & $302(19.3)$ & $120(11.9)$ & $<0.001$ \\
\hline Urinary infection & $217(8.4)$ & $91(5.8)$ & $126(12.5)$ & $<0.001$ \\
\hline mRS at discharge & $4.0(2.0-5.0)$ & $4.0(2.0-05.0)$ & $4.0(2.0-5.0)$ & 0.008 \\
\hline $\mathrm{mRS}>2$ at discharge & $1690(68.5)$ & $990(65.9)$ & $700(72.6)$ & $<0.001$ \\
\hline Death & $393(15.3)$ & $237(15.1)$ & $156(15.5)$ & 0.779 \\
\hline Hospital length & $7.0(4.0-12.0)$ & $7(4.0-12.0)$ & $7(5.0-13.0)$ & 0.037 \\
\hline
\end{tabular}

Multivariate analysis. Predictors of poor prognosis

\begin{tabular}{|c|c|c|c|}
\hline & OR & $95 \% \mathrm{Cl}$ & p-value \\
\hline Age & 1.05 & $1.04-1.05$ & $<0.001$ \\
\hline \multicolumn{4}{|l|}{ Age groups } \\
\hline - $\quad 18-39$ & 1.56 & $1.41-1.72$ & $<0.001$ \\
\hline - $\quad 40-49$ & 1.19 & $0.62-2.27$ & 0.609 \\
\hline - $\quad 50-59$ & 2.25 & $1.24-4.09$ & 0.008 \\
\hline $\begin{array}{l}-\quad 60-69 \\
-1\end{array}$ & 2.82 & $1.58-5.02$ & $<0.001$ \\
\hline - $\quad 70-79$ & 4.43 & $2.51-7.82$ & $<0.001$ \\
\hline - $\quad 80-89$ & 7.98 & $4.47-14.22$ & $<0.001$ \\
\hline - $\quad>=90$ & 14.4 & $5.77-36.00$ & $<0.001$ \\
\hline \multicolumn{4}{|l|}{ Baseline characteristics } \\
\hline Sex female & 1.32 & $1.04-1.67$ & 0.025 \\
\hline Alcoholism & 1.32 & $0.79-2.21$ & 0.283 \\
\hline Smoker & 1.32 & $0.79-2-20$ & 0.288 \\
\hline Hypertension & 0.98 & $0.76-1.55$ & 0.884 \\
\hline Diabetes mellitus & 1.37 & $1.04-1.81$ & $<0.001$ \\
\hline Atrial fibrillation & 1.25 & $0.80-1.98$ & 0.331 \\
\hline Ischemic heart disease & 0.86 & $0.55-1.33$ & 0.490 \\
\hline \multicolumn{4}{|l|}{ Previous treatment } \\
\hline Anticoagulants & 1.26 & $0.82-1.94$ & 0.292 \\
\hline Antiplatelets & 1.18 & $0.88-1.58$ & 0.280 \\
\hline Statins & 1.11 & $0.85-1.44$ & 0.453 \\
\hline Stroke awakening & 1.27 & $0.94-1.70$ & 0.120 \\
\hline \multicolumn{4}{|l|}{ Stroke severity } \\
\hline NIHSS at admission & 1.30 & $2.0(1.0-4.0)$ & $<0.001$ \\
\hline \multicolumn{4}{|l|}{ Etiology } \\
\hline Primary & 0.86 & $0.64-1.15$ & 0.316 \\
\hline \multicolumn{4}{|l|}{ Clinical evolution } \\
\hline Febrile syndrome & 2.90 & $2.06-4.08$ & $<0.001$ \\
\hline
\end{tabular}

